\title{
UNA DOPPIA REGOLARITÀ NEL SISTEMA SOLARE
}

\author{
Nota del Dott. Marianr, Pierugci
}

\section{Introduzione.}

$\S 1$.

Ancor vivente il Laplace era noto come i primi tre satelliti di Giove siano soggetti ad una regolarità che ne lega i periodi di rivoluzione.

In seguito, nel modo (apparentemente disordinato) con cui sono distribuiti i pianeti ed i satelliti, si è cercato con. insistenza di scoprire un ordinamento abbastanza semplice; dal quale, risalendo poi dal conosciuto all' incognito, poter sapere qualche cosa sulla formazione del nostro sistema solare.

Del resto già un secolo e mezzo addietro la celebre legge empirica Titius-Bode aveva alquanto coordinato le conoscenze allora esistenti sulla distribuzione delle distanze dei pianeti dal sole; parve anzi che rispondesse ad una legge reale, sconosciuta; specialmente allorquando, con la scoperta dei primi asteroidi, si potè osservare che venivano a colmarsi delle lacune, considerate dapprima come un difetto della regola stessa. Se non che, trovato un nuovo pianeta, Nettuno, fu ncessario abbandonarla per accoglierne altre, dello stesso tipo, enunciate successivamente da Wurm, da Gaussin, da Belot, ecc. Tutte queste regole, con due o più parametri, contengono un termine esponenziale.

Pochi anni fa ') l' Armellini ba enunciato una legge esponenziale, con un solo parametro,

$$
a_{n}=1,53^{n} \quad \text { (distanza terra - sole }=1 \text { ) }
$$

dove $a_{n}$ è la distanza media del pianeta ennesimo dal sole.

1) Atti della R. Acc. dei Lincei, 1917, 1.o Semestre, pag. 316. 
Tale regola è superiore a quelle precedentemente enunciate : $10^{\circ}$ per essere la più semplice; $20^{\circ}$ per possedere una sola lacuna; $3 .^{\circ}$ per avere degli scarti il cui error medio è minore di quelli corrispondenti alle altre regole.

Poco dopo ") il Prof. Burgatti estese la regola ai satelliti ; o, per meglio dire, trovò che anche per i satelliti valgono, caso per caso, delle regole esponenziali mono-parametriche del tipo

$$
a_{n}=k^{n}
$$

dove $k$ è un parametro che cambia da un sistema all' altro.

Il fatto che tutte queste regole contengano un termine esponenziale suggerisce 1 ' ipotesi che la forma esponenziale traduca analiticamente una qualche causa, magari ancor sconosciuta, la cui ricerca però ei si presenta tutt'altro che facile. Il nostro compito potrebbe, forse, divenire più semplice se si arrivasse a trasformare l' espressione analitica in maniera da ottenerne un' altra molto semplice tra grandezze di significato fisico o geometrico ben definito, nella quale non entrassero affatto dei valori medî. A tale trasformazione, come vedremo, si può giungere assai facilmente.

Se non che da qualche anno sono state enunciate due nuove regole empiriche che non contengono affatto dei termini esponenziali.

La prima è quella che io proposi nel $\left.1919^{\circ}\right)$. « Le distanze medie dei pianeti dal sole $\theta$ dei satelliti dal loro pianeta. crescono come la serie naturale dei numeri ».

La seconda fu enunciata recentemente dal Prof. Dittrich ${ }^{3}$ ) ed è espressa, con le solite notazioni, dalla formula

$$
a_{n}=k n^{2} \text {. }
$$

Quest' ultima è applicabile a 33 corpi del nostro sistema solare; la sua portata è quindi assai vasta; ma secondo il

1) Memorie dell' Accademia di Bologna.

2) Il Nuoro Cimento, 1919, pag. 107, 2..$^{\circ}$ Semestre.

$\left.{ }^{3}\right)$ Astronomische Nachrichten. 1.0 Giugno 1921, N. 5113, Vol. 214. 
mio parere ha il difetto di possedere troppe lacune (più dei posti realmente occupati); benchè, a dire il vero, il genere della regola non implichi necessariamente che tutti i posti debbano essere occupati, ma piuttosto che i posti effettivamente occupati non possono che trovarsi fra certi deierminati. D' altra parte però questa ultima osservazione vale anche per la mia regola, che ha 13 lacune contro 36 posti effettivamente occupati, pur possedendo un error - medio più basso di quello della regola Dittrich.

La nostra trasformazione dovrebbe tentarsi quindi anche per il secondo tipo di regole; ma sorge ora la questione pregiudiziale: possono coesistere due tipi diversi di regole empiriche, se queste regole stesse corrispondono a qualche legge effettiva? Questa pregiudiziale, che a prima giunta sembrerebbe impedire del tutto ogni passo ulteriore della ricerca, vedremo però che non ha valore alcuno; poichè, partendo dalle due regole più esatte dei due tipi, (quella di Armellini e la mia) si arriva a due formule semplici, conteneuti grandezze fisiche e geometriche ben definite, che possono coesistere perfettamente.

\section{$\S 2$.}

\section{Le due regolarità coesistenti.}

La regola non parametrica (come potremo chiamarla in seguito) da me proposta per le distanze medie dei pianeti e di alcune comete dal sole e dei satelliti dal loro pianeta è già stata implicitamente trasformata, nel modo che cercavamo, in alcune mie precedenti note $\left.{ }^{1}\right)$. Difatti si arrivò all' eunnciato più generale. I raggi equivalenti o dei pianeti, dei satelliti di uno stesso sistema e delle comete di una classe

1) Il Nuovo Cimento, 1920, 1. ${ }^{\circ}$ Semestre, pag. 31.

id. $\quad 1921,10^{\circ}$ Stmestre, id. 259 e 263.

id. $\quad, 1932,10^{\circ}$ Semestre, pagg, 227, 233, 333. 
crescono come numeri interi, dove per raggio equivalente $p$ si intendeva

$$
p=\sqrt{a b}
$$

essendo $a$ e $b$ i semiassi dell' ellisse.

Dunque, scegliendo opportunamente l' unità di misura, si può scrivere

$$
p_{n}=n \text {. }
$$

Ma si può subito trasformare la (4), introducendo l' area $A_{n}$ della ellisse descritta dal corpo ennesimo

e si ottiene

$$
A_{n}=\pi \sqrt{a b} \Rightarrow \pi \rho^{2}
$$

$$
\mathrm{A}_{n}=\pi n^{2}
$$

o ancora (scegliendo in modo conveniente l' unità)

$$
\mathrm{A}_{n}=n^{2} \text {. }
$$

Pure nei già citati lavori si era in qualche modo giunti ad una relazione trasformata delle regole esponenziali.

Difatti ivi mostrai come la regola Armellini migliorasse alquanto (diminuisce l' error - medio relativo) col modificare leggermente il parametro, facendo cioè

$$
a_{n}=1,54^{n} ;
$$

ed osservai come il valore 1,54 sia abbastanza vicino a

$$
\sqrt[3]{2^{2}}(=1,5874) ;
$$

(ed anzi sarebbe facile vedere come si otterrebbe ancora qualche vantaggio ponendo nella (7) una terza cifra decimale, in modo da avere un parametro compreso tra 1,54 e 1,55 uguale a $\sqrt{1,92^{2}}$ ossia 1,544$)$.

Se valesse la

$$
a_{n}=1,5874^{n}
$$

potremmo anche scrivere (per la 3. Legge di Kepler)

$$
\mathrm{T}_{n}=2^{n}
$$


(indicando con $\mathrm{T}_{n}$ il periodo di rivoluzione del pianeta ennesimo), pur di scegliere in modo opportuno l' unità di tempo.

Ma appunto la (9) è valida per $i$ cinque satelliti interni di Giove e per $i$ due di Marte (vedi l' ultima delle note citate) ed anche per $i$ cinque sate? iti esterni di Saturno (come mostra la tabella seguente, in cur sono posti i dati dell'Annuario del Bureau des Longitudes del 1920).

\begin{tabular}{|c|c|c|c|}
\hline Satelliti & $a_{n}$ osservati & $a_{n}$ calcolati & $\begin{array}{l}\text { errore } \\
\text { relativo }\end{array}$ \\
\hline Rea & 8,78 & $9,00=9,00 \times 1,5874^{\circ}$ & $3,11 \%$ \\
\hline $\begin{array}{l}\text { Titano, Temi, } \\
\text { Iperione }\end{array}$ & 22,35 & $22,41=9,00 \times 1,5874^{2}$ & $0,27 \%$ \\
\hline & $\begin{array}{l}\text { [media dei valori } \\
\text { estremi : } \\
\quad 20,22-24,49 \text { | }\end{array}$ & & \\
\hline Giapeto & 58,91 & $55,80=9,00 \times 1,5874^{4}$ & $5,59 \%$ \\
\hline \multirow[t]{2}{*}{ Febo } & 214,4 & $223,2=9,00 \times 1,5874^{7}$ & $4,10 \%$ \\
\hline & & error - medio : & $3,87 \%$ \\
\hline
\end{tabular}

ì quasi superfluo osservare che la costante 9,00 potrebbe eliminarsi prendendo come unità di misura, invece della lunghezza del semidiametro equatoriale del pianeta (come si è fatto qui), una lunghezza nove volte maggiore.

Per i tre casi dianzi accennati siamo quindi giunti ad ottenere una forma della regola in cui non entrano valori medî, ma solo il periodo di rivoluzione ed un parametro semplicissimo : 2 . 
Nel caso dei pianeti, invece, si potrebbe interpretare la regola Armellini (7) così : i pianeti hanno una certa tendenza a raggiungere una posizione espressa appunto dalla (8). I satelliti di Urano soddisfano pure ad una relazione esponenziale, con un parametro ancora approssimato per difetto a 3 $\sqrt{2^{2}}$. Vale per essi la relazione $: a^{n}=1,50^{n}$, come mostra il quadro che segue.

\begin{tabular}{l|r|r|c}
\hline Satelliti & $\begin{array}{c}a_{n} \\
\text { osservati }\end{array}$ & $a_{n}$ calcolati & errore relativo \\
\hline Ariel & 7,71 & $7,20=7,20 \times 1,50^{\circ}$ & $6,61 \%$ \\
Umbriel & 10,75 & $10,80=7,20 \times 1,50^{1}$ & $0,47 \%$ \\
Titania & 17,63 & $16,20=7,20 \times 1,50^{2}$ & $8,83 \%$ \\
Oberon & 23,57 & $24,30=7,20 \times 1,50^{3}$ & $3,00 \%$ \\
& & error - medio : $\mathbf{5 , 6 6 \%}$
\end{tabular}

Potremmo dunque concludere : $i$ pianeti e gran parte aei satelliti sono dirposti - o tendono a disporsi - in modo da soddisfare $l a$

$$
\mathrm{T}_{n}=2^{n}
$$

o, se si vuole, la

$$
\frac{\mathrm{T}_{n+1}}{\mathrm{~T}_{n}}=2
$$

Ma è facile vedere che anche per i due casi esclusi (satelliti esterni di Giove e satelli interni di Saturno) vale una relazione analoga

$$
\frac{\mathrm{T}_{n+1}}{\mathrm{~T}_{n}}=k
$$


dove $k$ è un rapporto molto semplice (in generale però diverso dal rapporto: 2/1) che può variare col variare di $n$. Ciò si vede nella tabella seguente:

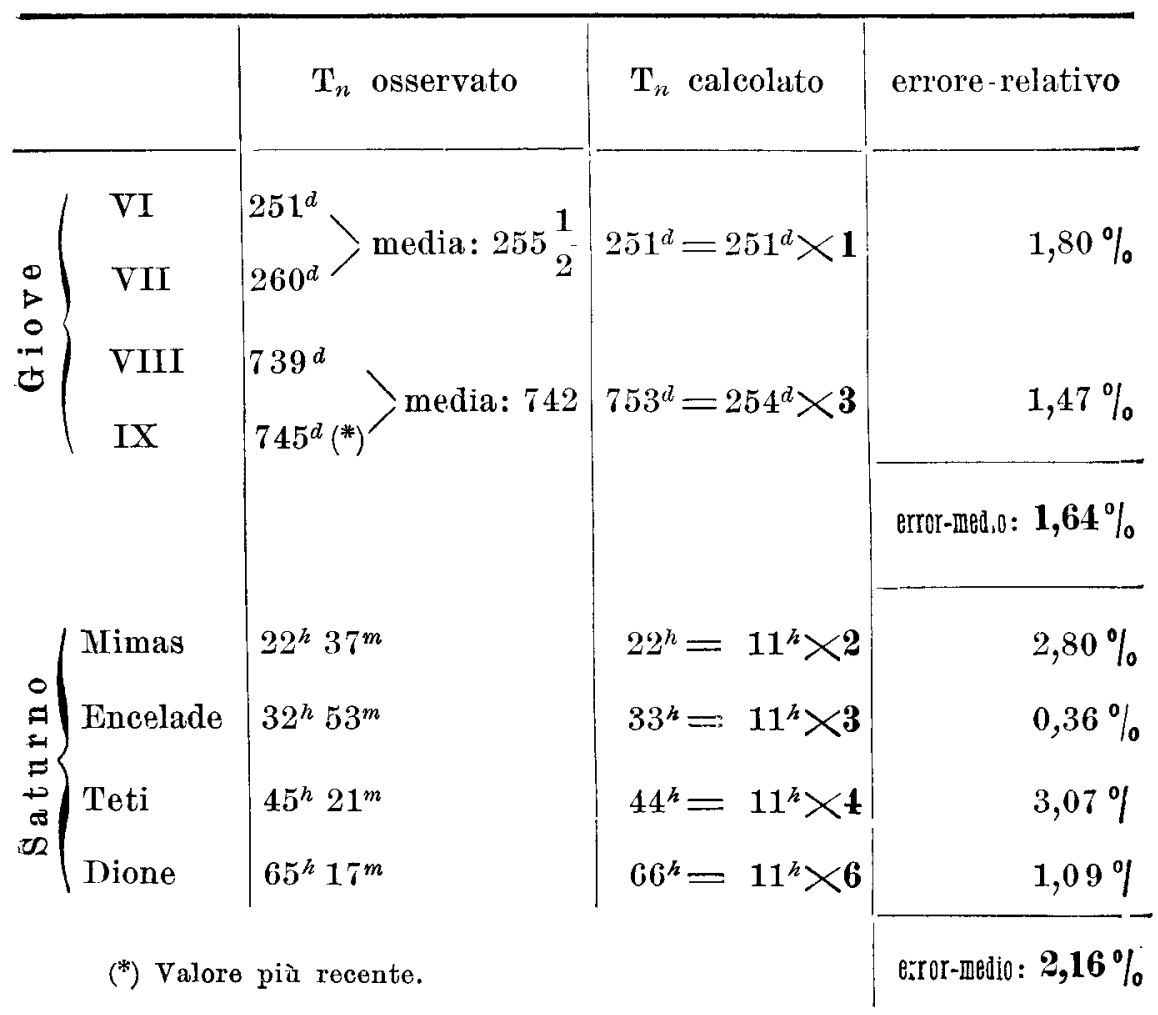

$\S 3$.

Siamo così arrivati nei due tipi di regole (12 esponenziale monoparametrica; $2^{\mathbf{a}}$ non parametrica), o meglio, nelle regole più esatte di ciascun tipo, a sbarazzarci di valori medî; di modo che, riepilogando, possiamo enunciare le due regole seguenti, nelle quali non compaiono che elementi geometricamente o fisicamente ben definiti :

1) Le aree racchiuse nelle orbite dei pianeti, dei satelliti e di alcune comete crescono come $i$ quadrati dei numeri interi. 
2) I periodi di rivoluzione stanno tra di loro in rapporti razionali semplici; anzi, nella maggior parte dei casi $i$ periodi di corpi celesti contigui sono posti - (o tendono a porsi) - nel rapporto semplicissimo 1/2.

Esprimendo la 2. ${ }^{a}$ nella forma più ristretta (non sempre valida) otteniamo le due relazioni (di forma suggestiva se considerate I' una in confronto dell' altra)

$$
\begin{aligned}
& \mathrm{A}_{n}=n^{2} \\
& \mathrm{~T}_{n}=2^{n} .
\end{aligned}
$$

Credo opportuno riportare in una tavola unica gli errori relativi di ciascuna regola nei varî sistemi e le lacune; affinchè il lettore non sia costretto a riandare tutti gli specchietti delle precedenti note, già citate.

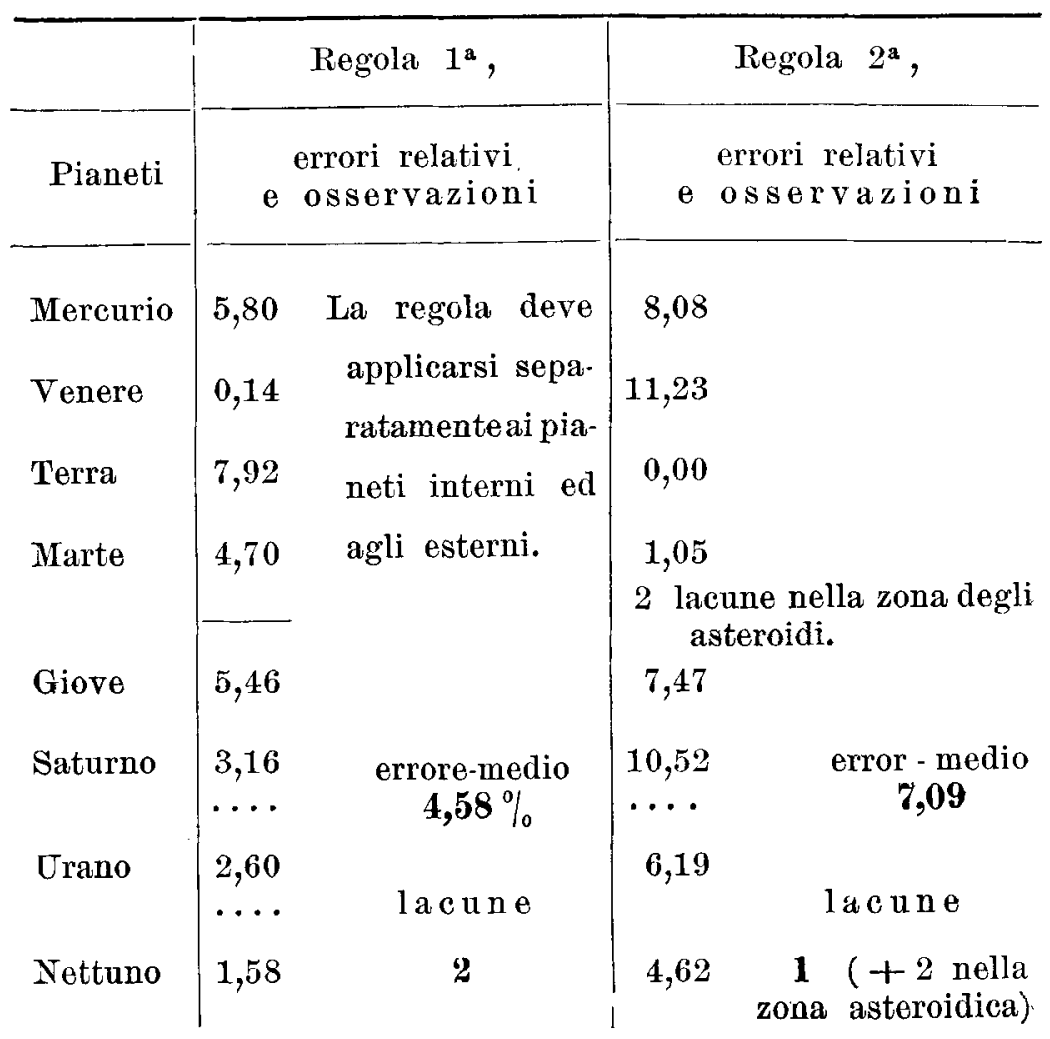




\begin{tabular}{|c|c|c|c|c|}
\hline & & Regola $1^{\mathrm{a}}$, & \multicolumn{2}{|c|}{ Regola $2^{\mathrm{a}}$, } \\
\hline $\begin{array}{l}\text { Satelliti } \\
\text { di } \text { Marte }\end{array}$ & & $\begin{array}{l}\text { rori relativi } \\
\text { servazioni }\end{array}$ & $\begin{array}{ll} & e \\
e & 0\end{array}$ & $\begin{array}{l}\text { i relativi } \\
\text { 3rvazioni }\end{array}$ \\
\hline $\begin{array}{l}\text { Phobos } \\
\text { Deimos }\end{array}$ & $\begin{array}{l}\cdots, 36 \\
0,36 \\
0,00\end{array}$ & $\begin{array}{c}\text { error - medio } \\
\mathbf{0 , 2 6} \% \\
\text { lacune } \\
\mathbf{3}\end{array}$ & $\begin{array}{c}0,36 \\
\ldots \\
0,00\end{array}$ & $\begin{array}{c}\text { error - medio } \\
\mathbf{0 , 2 6} \% \\
\text { lacune } \\
\mathbf{1}\end{array}$ \\
\hline $\begin{array}{l}\text { Satelliti } \\
\text { di Giove }\end{array}$ & & & & \\
\hline 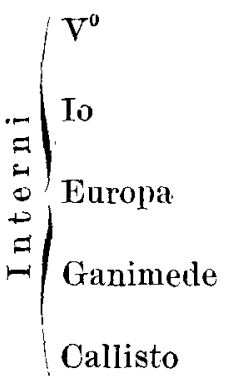 & $\begin{array}{l}14,76 \\
8,74 \\
\\
2,97 \\
\ldots \\
2,37\end{array}$ & $\begin{array}{c}\text { error - medio } \\
8,78 \\
\text { lacune } \\
1\end{array}$ & $\begin{array}{l}2,84 \\
\ldots \ldots \\
4,51 \\
4,47 \\
4,03 \\
6,29\end{array}$ & $\begin{array}{c}\text { error - medio } \\
4,58 \\
\begin{array}{c}\operatorname{lacune} \\
1\end{array}\end{array}$ \\
\hline \begin{tabular}{l|l}
$\mathrm{VI}^{0}$ \\
- \\
$\mathrm{VII}^{0}$ \\
$\dot{\Phi}$
\end{tabular} & 0,15 & $\begin{array}{c}\text { error-medio } \\
\mathbf{0 , 1 5} \% \\
\text { lacune } \\
\mathbf{0}\end{array}$ & $\begin{array}{l}1,80 \\
\ldots \\
1,47\end{array}$ & $\begin{array}{c}\text { error - medio } \\
1,64 \% \\
\text { lacune } \\
1\end{array}$ \\
\hline
\end{tabular}




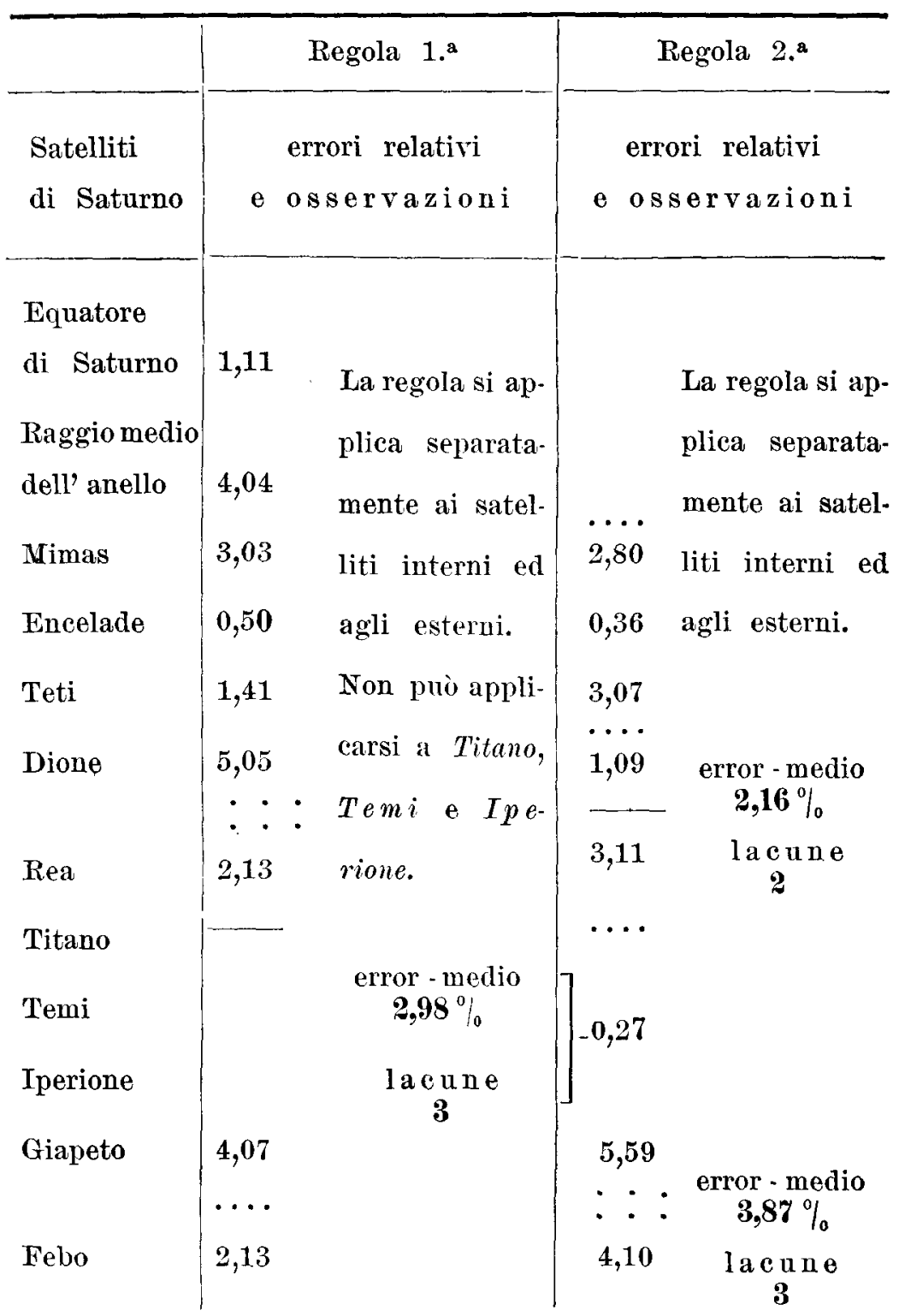




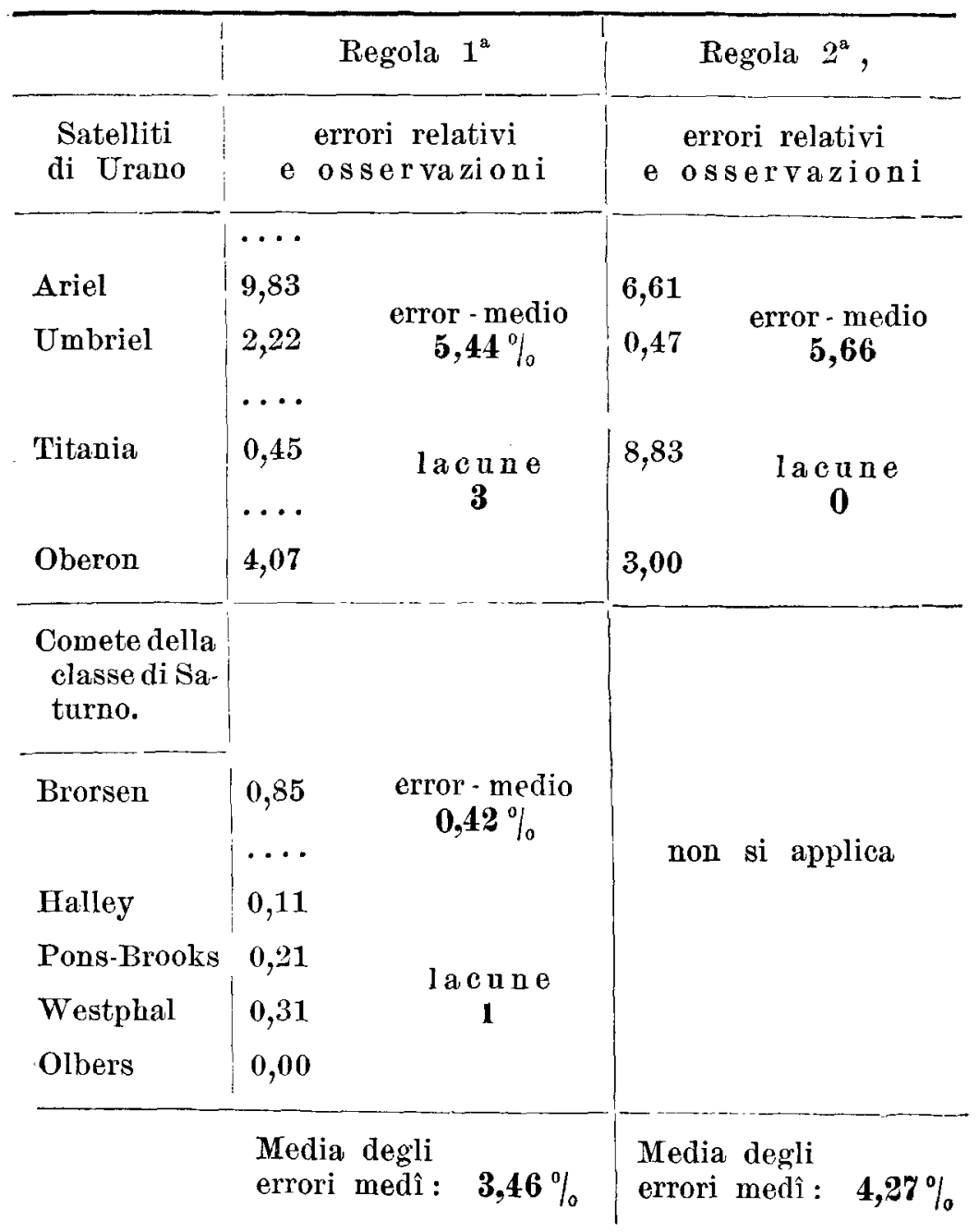

La $1^{\mathrm{a}}$, contiene dunque 13 lacune contro 36 posti effettivamente occupati; la $2^{2} 13$ lacune contro 33 posti pieni (2 lacune possono però considerarsi come occupate dagli asteroidi).

La media degli errori medî è fatta dando ad ogni singolo error-medio un peso uguale al numero dei corpi del sistema a cai si riferisce (ad es. il peso 8 per i pianeti, il peso 4 
per i satelliti di Urano); tale media, come si vede, è di $3,46 \%$ per la $1^{\mathrm{a}}$ e $4,27 \%$ per la seconda regola.

La $1^{a}$ sarebbe dunque leggermente migliore della $2^{a}$; anche perchè la $1^{a}$ regola há un enunciato uniforme e netto in tutti i sistemi.

In ogni modo, però, il fatto più saliente è che esse possano coesistere. $\mathbf{E}$ come ciò possa accadere si comprende facilmente quando si pensi che sarebbe sempre possibile soddisfarle senza scarti e senza lacune, pur di disporre in modo conveniente delle eccentricità; e che, d'altra parte, esse, in realtà, sono soddisfatte con lacune e scarti, i quali rendono possibile la loro coesistenza, anche quando le orbite sono quasi circolari.

È pure notevole che l'enunciato più ristretto della seconda regola espresso dalla

$$
a_{n}=1,5874^{n}
$$

è sempre soddisfatto in quei casi in cui la prima regola è applicabile malamente (ad esempio per $i$ satelliti interni di Giove) o non vale affatto (satelliti esterni di Saturno); come pure la prima regola vale molto bene in quei casi nei quali l'enunciato più ristretto $(8)$ della seconda non può applicarsi (per esempio per i satelliti interni di Saturno e per gli esterni di Giove).

Così che possiamo concludere che vale sempre una delle due relazioni semplici (6) e (9) sopra considerate. Con queste due relazioni si potrebbe esaurire tutto il sistema solare, con 40 posti effettivamente occupati, formando così come una regola mista; la cosa è mostrata dal quadro che segue: 


\begin{tabular}{|c|c|c|c|c|}
\hline & Regola & $\begin{array}{c}\text { Posti } \\
\text { occupati }\end{array}$ & Lacune & Error-medio \\
\hline Pianeti $\cdot \cdot \cdot \cdot \cdot \cdot \cdot \cdot$ & $(6)$ & 8 & 2 & $4.58 \%$ \\
\hline Satelliti di Marte $\cdot$ • . & (9) & 2 & 1 & $0,26 \%$ \\
\hline$\gg$ di Giove interni & (9) & 5 & 1 & $4,58 \%$ \\
\hline$》 \quad$ esterni & $(6)$ & 4 & 0 & $0,15 \%$ \\
\hline » di Saturno interni & $(6)$ & $\left.6^{\prime}\right)$ & $\mathbf{0}$ & $3,01 \%$ \\
\hline$\gg \quad$ esterni & (9) & 6 & 4 & $3,09 \%$ \\
\hline$\gg$ di Urano . . . & $(6)$ & 4 & 3 & $5,44 \%$ \\
\hline $\begin{array}{c}\text { Comete della classe di Sa- } \\
\text { turno } \cdot \cdot^{-} \cdot \cdot^{-} \cdot\end{array}$ & (6) & 5 & 1 & $0,41 \%$ \\
\hline
\end{tabular}

Media degli errori-medî : $\mathbf{3 , 0 2} \%$

Ciò non significa che sia opportuno assumere una regola mista, ma soltanto serve a farci vedere meglio come sia sempre ben soddisfatta una delle due: la (6) o la (9).

Qui giunti ci si presenta ancora difficile decidere se le due regole siano da attribuirsi ad una sola causa, o a due separate; e se questa o queste cause siano già conosciute o ancora da trovarsi. Benchè a prima vista sembri più probabile l'ipotesi delle due cause, io preferisco fermarmi a questo punto, senza nulla aggiungere agli enunciati semplici esposti precedentemente.

\section{Conelusione.}

Partendo dalle regole empiriche sulle distanze medie dei pianeti dal sole sino ad oggi conosciute, siamo giunti ad enunciare le due proposizioni :

1) Contano come satelliti l'equatore e la zona centrale dell'anello di Saturno. 
1. - Le aree racchiuse nelle orbite dei pianeti, dei satelliti di quasi tutti i sistemi, e di alcune comete, erescono come i quadrati dei numeri interi.

2. - I periodi di rivoluzione stanno tra di loro in rapporti razionali semplici; anzi, nella maggior parte dei casi, $\mathbf{i}$ periodi di corpi celesti contigni sono posti (o tendono a porsi) nel rapporto semplicissimo $1: 2$.

Vale poi sempre una almeno delle due relazioni

$$
\mathrm{A}_{n}=n^{\mathrm{a}} \quad \mathrm{T}_{n}=2^{n}
$$

(dove $A_{n}$ e $\mathrm{T}_{n}$ sono l'area dell'orbita e il periodo di rivoluzione appartenenti al corpo ennessimo) nelle quali i secondi membri si ottengono l'uno dall'altro scambiando la base con l'esponente.

Siamo dunque in presenza di relazioni semplici, contenenti, non più valori medî, ma grandezze fisicamente e geometricamente ben definite; dalle quali sembra piì agevole poter tentare una qualche interpretazione della cosmogonia relativa al nostro sistema solare.

In ogni modo è mostrato come sia preferibile, in seguito, fissare lo studio sulle aree e su i periodi di rivoluziope, piuttosto che sulle distanze medie dei pianeti, dei satelliti e delle comete dai loro centri attrattiri.

Pisa, Istituto Fisico della R. Università. 